\title{
The Kirchhoff Index of Folded Hypercubes and Some Variant Networks
}

\author{
Jiabao Liu, ${ }^{1,2,3}$ Xiang-Feng Pan, ${ }^{1}$ Yi Wang, ${ }^{2}$ and Jinde Cao ${ }^{2,4}$ \\ ${ }^{1}$ School of Mathematics Science, Anhui University, Hefei 230601, China \\ ${ }^{2}$ Department of Mathematics, Southeast University, Nanjing 210096, China \\ ${ }^{3}$ Anhui Xinhua University, Hefei 230088, China \\ ${ }^{4}$ Department of Mathematics, Faculty of Science, King Abdulaziz University, Jeddah 21589, Saudi Arabia
}

Correspondence should be addressed to Jinde Cao; jdcao@seu.edu.cn

Received 3 November 2013; Accepted 25 November 2013; Published 16 January 2014

Academic Editor: Chuangxia Huang

Copyright ( 2014 Jiabao Liu et al. This is an open access article distributed under the Creative Commons Attribution License, which permits unrestricted use, distribution, and reproduction in any medium, provided the original work is properly cited.

\begin{abstract}
The $n$-dimensional folded hypercube $F Q_{n}$ is an important and attractive variant of the $n$-dimensional hypercube $Q_{n}$, which is obtained from $Q_{n}$ by adding an edge between any pair of vertices complementary edges. $F Q_{n}$ is superior to $Q_{n}$ in many measurements, such as the diameter of $F Q_{n}$ which is $\lceil n / 2\rceil$, about a half of the diameter in terms of $Q_{n}$. The $\operatorname{Kirchhoff}$ index $\operatorname{Kf}(G)$ is the sum of resistance distances between all pairs of vertices in $G$. In this paper, we established the relationships between the folded hypercubes networks $F Q_{n}$ and its three variant networks $l\left(F Q_{n}\right), s\left(F Q_{n}\right)$, and $t\left(F Q_{n}\right)$ on their Kirchhoff index, by deducing the characteristic polynomial of the Laplacian matrix in spectral graph theory. Moreover, the explicit formulae for the Kirchhoff indexes of $F Q_{n}, l\left(F Q_{n}\right), s\left(F Q_{n}\right)$, and $t\left(F Q_{n}\right)$ were proposed, respectively.
\end{abstract}

\section{Introduction}

It is well known that interconnection networks play an important role in parallel communication systems. An interconnection network is usually modelled by connected graphs $G=(V, E)$, where $V$ denotes the set of processors and $E$ denotes the set of communication links between processors in networks. In this work, we are concerned with finite undirected connected simple graphs (networks). For the graph theoretical definitions and notations, we follow [1].

The adjacency matrix $A(G)$ of $G$ is an $n \times n$ matrix with the $(i, j-)$ entry equal to 1 if vertices $i$ and $j$ are adjacent and 0 otherwise. Suppose that $D(G)=\operatorname{diag}\left(d_{1}(G), d_{2}(G), \ldots, d_{n}(G)\right)$ is the degree diagonal matrix of $G$, where $d_{i}(G)$ is the degree of the vertex $i, i=1,2, \ldots, n$. Let $L(G)=D(G)-A(G)$ be called the Laplacian matrix of $G$. Then, the eigenvalues of $A(G)$ and $L(G)$ are called eigenvalues and Laplacian eigenvalues of $G$, respectively. For more details, we refer to [1].

Let $G$ be a graph with vertices labelled $1,2, \ldots, n$. The resistance distances between vertices $i$ and $j$, denoted by $r_{i j}$, are defined to be the effective electrical resistance between them if each edge of $G$ is replaced by a unit resistor [2]. A famous distance-based topological index as the Kirchhoff index $\operatorname{Kf}(G)$, is defined as the sum of resistance distances between all pairs of vertices in $G$. Define

$$
\operatorname{Kf}(G)=\frac{1}{2} \sum_{i=1}^{n} \sum_{j=1}^{n} r_{i j}(G),
$$

known as the Kirchhoff index of $G$ [2].

The Kirchhoff index attracted extensive attention due to its wide applications in physics, chemistry, graph theory, and so forth [3-6]. For example, Zhu et al. [7] and Gutman and Mohar [8] proved that relations between Kirchhoff index of a graph and Laplacian eigenvalues of the graph. The Kirchhoff index also is a structure descriptor [9].

However, it is difficult to design some algorithms $[7,10,11]$ to calculate resistance distances and the Kirchhoff indexes of graphs. Hence, it makes sense to find explicit closed form 
for some special classes of graphs, for instance, the Kirchhoff index for cycles and complete graphs [12] which has been computed, geodetic graphs [13], some composite graphs [14], and composite networks [15]. Besides, many efforts were also made to obtain the Kirchhoff index bounds for some graphs $[11,16]$ and characterize extremal graphs as well, such as bicyclic graphs and cacti graphs $[6,17]$. Details on its theory can be found in recent papers $[11,16,17]$ and the references cited therein.

The hypercube $Q_{n}$ is one of the most popular and efficient interconnection networks due to its excellent performance for some practical applications. There is a large amount of literature on the properties of hypercubes networks [18-20].

As an important variant of $Q_{n}$, the folded hypercube networks $F Q_{n}$, proposed by El-Amawy and Latifi [18], are the graph obtained from $Q_{n}$ by adding an edge between any pair of vertices complementary addresses. The folded hypercube $F Q_{n}$, in which diameter of $F Q_{n}$ is $\lceil n / 2\rceil$, about half the diameter of $Q_{n}$, has the same number of vertices as a hypercube $Q_{n}$ and $2^{n-1}$ edges more than hypercube; at the same time, the folded hypercubes preserve the symmetric properties of the hypercubes. The folded hypercubes $F Q_{n}$ obtained considerable attention due to its perfect properties, such as symmetry, regular structure, strong connectivity, and small diameter, and many of its properties have been explored [21-26].

But few works appear on the Kirchhoff index for the combinatorial networks, such as hypercubes $Q_{n}$ and folded hypercubes $F Q_{n}$, except that Palacios and Renom [11] studied the bounds of the Kirchhoff index of hypercubes $Q_{n}$ by using probabilistic tools in 2010. In present paper, we established the relationships between the folded hypercubes networks $F Q_{n}$ and three variant networks $l\left(F Q_{n}\right), s\left(F Q_{n}\right)$, and $t\left(F Q_{n}\right)$ on their Kirchhoff index, by deducing the characteristic polynomial of the Laplacian matrix in spectral graph theory.

Recall the definitions of $\mathrm{n}$-dimensional folded hypercubes networks $F Q_{n}$ as follows [18].

Definition 1 (see [18]). The folded hypercubes $F Q_{n}$ can be constructed from $Q_{n}$ by adding an edge to every pair of vertices with complementary addresses. Two vertices $X=$ $x_{1} x_{2} \cdots x_{n}$ and $\bar{X}=\overline{x_{1}} \overline{x_{2}} \cdots \overline{x_{n}}$ are adjacent in the folded hypercubes $F Q_{n}$.

From above definition of $F Q_{n}$, it is easy to get that the folded hypercubes $F Q_{n}$ have $2^{n}$ vertices and $(n+1) 2^{n-1}$ edges, respectively.

The line graph of a graph $G$, denoted by $l(G)$, is the graph whose vertices correspond to the edges of $G$ with two vertices of $L(G)$ being adjacent if and only if the corresponding edges in $G$ share a common vertex. The subdivision graph of a graph $G$, denoted by $s(G)$, is the graph obtained by replacing every edge in $G$ with a copy of $P_{2}$ (path of length two). The total graph of a graph $G$, denoted by $t(G)$, is the graph whose vertices correspond to the union of the set of vertices and edges of $G$, with two vertices of $t(G)$ being adjacent if and only if the corresponding elements are adjacent or incident in $G$.

Gao et al. [27] obtained special formulae for the Kirchhoff index of $l(G), s(G)$, and $t(G)$, where $G$ is a regular graph. Motivated by above results, we present the corresponding calculated formulae for the Kirchhoff index of the hypercubes networks $Q_{n}$ and its three-variant networks $l\left(F Q_{n}\right), s\left(F Q_{n}\right)$, and $t\left(F Q_{n}\right)$ in this paper.

The remainder of present paper is organized as follows. Section 2 gives some basic notations and some preliminaries in our discussion. The proofs of our main results are in Section 3 and some conclusions are given in Section 4, respectively.

\section{Notations and Some Preliminaries}

In this section, we introduced some basic properties which we need to use in the proofs of our main results.

M. Chen and B. X. Chen have studied the Laplacian spectra of folded hypercubes networks in 2011 [21].

Lemma 2 (see [21]). For the folded hypercubes networks $F Q_{n}$ with $n \geq 2$, the spectrum of Laplacian matrix in terms of hypercubes networks is as follows.

(1) If $n \equiv 0(\bmod 2)$,

$$
\operatorname{Spec}\left(F Q_{n}\right)=\left(\begin{array}{ccccccc}
0 & 4 & 8 & \cdots & 2 n-8 & 2 n-4 & 2 n \\
C_{n}^{n} & C_{n}^{n-1}+C_{n}^{n-2} & C_{n}^{n-3}+C_{n}^{n-4} & \cdots & C_{n}^{5}+C_{n}^{4} & C_{n}^{3}+C_{n}^{2} & C_{n}^{1}+C_{n}^{0}
\end{array}\right)
$$

(2) If $n \equiv 1(\bmod 2)$,

$$
\operatorname{Spec}\left(F Q_{n}\right)=\left(\begin{array}{ccccccc}
0 & 4 & 8 & \cdots & 2 n-6 & 2 n-2 & 2 n+2 \\
C_{n}^{0} & C_{n}^{1}+C_{n}^{2} & C_{n}^{3}+C_{n}^{4} & \cdots & C_{n}^{n-4}+C_{n}^{n-3} & C_{n}^{n-2}+C_{n}^{n-1} & C_{n}^{n}
\end{array}\right)
$$

where $C_{n}^{i}$ are the binomial coefficients; the elements in the first and second rows are the eigenvalues of the Laplacian matrix of folded hypercubes networks and the multiplicities of the corresponding eigenvalues.
Lemma 3 (see $[7,8])$. Let $G$ be a connected graph with $n \geq 2$ vertices; then,

$$
\mathrm{Kf}(G)=n \sum_{i=1}^{n-1} \frac{1}{\lambda_{i}}
$$


Let $P_{(G)}(x)$ be the characteristic polynomial of the Laplacian matrix of a graph $G$; the following results were shown in [27].

Lemma 4 (see [27]). Let $G$ be a r-regular connected graph with $n$ vertices and $m$ edges; then,

$$
\begin{gathered}
P_{l(G)}(x)=(x-2 r)^{m-n} P_{G}(x), \\
P_{s(G)}(x)=(-1)^{m}(2-x)^{m-n} P_{G}(x(r+2-x)), \\
P_{t(G)}(x)=(-1)^{m}(r+1-x)^{n} \\
\times(2 r+2-x)^{m-n} P_{G}\left(\frac{x(r+2-x)}{r-x+1}\right),
\end{gathered}
$$

where $P_{l(G)}(x), P_{s(G)}(x)$, and $P_{t(G)}(x)$ are the characteristic polynomial for the Laplacian matrix of graphs $l(G), s(G)$, and $t(G)$, respectively.

It is worthwhile to note that the conclusion of Lemma 4 is not completely correct; the authors [28] recently show the Laplacian characteristic polynomial of $t(G)$, where $G$ is a regular graph, which corrects Lemma 3 in Gao et al. [27] as follows.

Lemma 5 (see [28]). Let $G$ be a r-regular connected graph with $n$ vertices and $m$ edges; then,

$$
\begin{aligned}
& P_{s(G)}(x)=(-1)^{n}(2-x)^{m-n} P_{G}(x(r+2-x)), \\
& P_{t(G)}(x)= x(x-r-2)(x-2 r-2)^{m-n} \\
& \times \prod_{i=1}^{n-1}\left[\left(x^{2}-2 x-r x\right)+(3-2 x+r) \mu_{i}+\mu_{i}^{2}\right],
\end{aligned}
$$

where $P_{s(G)}(x), P_{t(G)}(x)$ are the characteristic polynomial for the Laplacian matrix of graphs $s(G)$ and $t(G)$, respectively.

The following lemma gives an expression on $\mathrm{Kf}(t(G))$ and $\mathrm{Kf}(G)$ of a regular graph $G$.

Lemma 6 (see [28]). Let $G$ be a r-regular connected graph with $n$ vertices and $m$ edges and $r \geq 2$; then,

$$
\begin{aligned}
\mathrm{Kf}(t(G))= & \frac{(r+2)^{2}}{2(r+3)} K f(G)+\frac{n^{2}\left(r^{2}-4\right)}{8(r+1)}+\frac{n}{2} \\
& +\frac{n(r+2)(r+4)}{2(r+3)} \sum_{i=1}^{n-1} \frac{1}{\mu_{i}+3+r} .
\end{aligned}
$$

For proving the formula for the Kirchhoff index on the subdivision graph of hypercubes $s\left(F Q_{n}\right)$, we prove the following lemma utilizing Vieta's theorem; in our proof, some techniques in [27] are referred.

Lemma 7. Let $P_{\left(F Q_{n}\right)}(x)$ be the characteristic polynomial of the Laplacian matrix of the folded hypercubes $F Q_{n}$ with $n \geq 2$ and

$$
P_{\left(F Q_{n}\right)}(x)=x^{2^{n}}+a_{1} x^{2^{n}-1}+a_{2} x^{2^{n}-2}+\cdots+a_{2^{n}-1} x ;
$$

then,

$$
\frac{\mathrm{Kf}\left(F Q_{n}\right)}{2^{n}}=-\frac{a_{2^{n}-2}}{a_{2^{n}-1}},
$$

where $a_{2^{n}-1}, a_{2^{n}-2}$ are the coefficient of $x$ and $x^{2}$ in the characteristic polynomial, respectively.

Proof. Let $\operatorname{Spec}\left(F Q_{n}\right)=\left\{\lambda_{0}, \lambda_{1}, \lambda_{2}, \ldots, \lambda_{n}, \lambda_{n+1}, \ldots, \lambda_{2^{n}-1}\right\}$. Then, $\lambda_{i}, i=1,2, \ldots, 2^{n}-1$, satisfy the following equation:

$$
x^{2^{n}-1}+a_{1} x^{2^{n}-2}+\cdots+a_{2^{n}-1}=0
$$

it is not difficult to check that $1 / \lambda_{i}, \quad i=1,2, \ldots, 2^{n}-1$, are the roots of equation

$$
a_{2^{n}-1} x^{2^{n}-1}+a_{2^{n}-2} x^{2^{n}-2}+\cdots+a_{1} x+1=0 .
$$

Note that $G$ is connected graph and the multiplicity of 0 as an eigenvalue of $L(G)$ is equal to the number of the connected components in $G$. So $a_{2^{n}-1} \neq 0$, by Lemma 3 and Vieta's theorem,

$$
\frac{\mathrm{Kf}\left(F Q_{n}\right)}{2^{n}}=\sum_{i=1}^{2^{n}-1} \frac{1}{\lambda_{i}}=-\frac{a_{2^{n}-2}}{a_{2^{n}-1}},
$$

where $a_{2^{n}-1}, a_{2^{n}-2}$ are the coefficient of $x$ and $x^{2}$ in the characteristic polynomial of the Laplacian matrix of the hypercubes $F Q_{n}$.

\section{Main Results}

3.1. The Kirchhoff Index in Folded Hypercubes Networks $F Q_{n}$. In this section, we firstly give formula for the Kirchhoff index of the folded hypercubes $F Q_{n}$ with any positive integer $n$.

Theorem 8. For the folded hypercubes networks $F Q_{n}$ with any positive integer $n$,

$$
\begin{aligned}
\text { (1) } \mathrm{Kf}\left(F Q_{n}\right) & =2^{n} \sum_{i=1}^{n / 2} \frac{C_{n}^{n-i}+C_{n}^{n-i-1}}{4 i}, \\
i=1,2, \ldots, \frac{n}{2}, \quad \text { if } n \equiv 0(\bmod 2) ; & \\
\text { (2) } \mathrm{Kf}\left(F Q_{n}\right) & =2^{n} \sum_{i=1}^{(n-1) / 2} \frac{C_{n}^{2 i-1}+C_{n}^{2 i}}{4 i}+\frac{2^{n-1}}{n+1}, \\
i & =1,2, \ldots, \frac{n-1}{2}, \quad \text { if } n \equiv 1(\bmod 2),
\end{aligned}
$$

where the $4 i, i=1,2, \ldots,(n-1) / 2$ or $n / 2$ are the eigenvalues of the Laplacian matrix of hypercubes networks $F Q_{n}$ and $C_{n}^{i}$ are the binomial coefficients, 
Proof. The hypercubes networks $F Q_{n}$ have $2^{n}$ vertices and $(n+1) 2^{n-1}$ edges.
By Lemma 3 , if $n \equiv 0(\bmod 2)$,

$$
\operatorname{Spec}\left(F Q_{n}\right)=\left(\begin{array}{ccccccc}
0 & 4 & 8 & \cdots & 2 n-8 & 2 n-4 & 2 n \\
C_{n}^{n} & C_{n}^{n-1}+C_{n}^{n-2} & C_{n}^{n-3}+C_{n}^{n-4} & \cdots & C_{n}^{5}+C_{n}^{4} & C_{n}^{3}+C_{n}^{2} & C_{n}^{1}+C_{n}^{0}
\end{array}\right)
$$

then

$$
\begin{aligned}
\mathrm{Kf}\left(F Q_{n}\right)= & 2^{n} \sum_{i=1}^{2^{n}-1} \frac{1}{\lambda_{i}} \\
=2^{n}( & \frac{C_{n}^{n-1}+C_{n}^{n-2}}{4 \times 1}+\frac{C_{n}^{n-3}+C_{n}^{n-4}}{4 \times 2}+\cdots \\
& \left.+\frac{C_{n}^{n-i}+C_{n}^{n-i-1}}{4 \times i}+\cdots+\frac{C_{n}^{1}+C^{0}}{4 \times(n / 2)}\right)
\end{aligned}
$$$$
\operatorname{Spec}\left(F Q_{n}\right)=\left(\begin{array}{ccccccc}
0 & 4 & 8 & \cdots & 2 n-6 & 2 n-2 & 2 n+2 \\
C_{n}^{0} & C_{n}^{1}+C_{n}^{2} & C_{n}^{3}+C_{n}^{4} & \cdots & C_{n}^{n-4}+C_{n}^{n-3} & C_{n}^{n-2}+C_{n}^{n-1} & C_{n}^{n}
\end{array}\right),
$$

By Lemma 3, if $n \equiv 1(\bmod 2)$, then

$$
\begin{aligned}
& \operatorname{Kf}\left(F Q_{n}\right) \\
& =2^{n} \sum_{i=1}^{2^{n}-1} \frac{1}{\lambda_{i}} \\
& =2^{n}\left(\frac{C_{n}^{1}+C_{n}^{2}}{4 \times 1}+\frac{C_{n}^{3}+C_{n}^{4}}{4 \times 2}+\cdots+\frac{C_{n}^{2 i}+C_{n}^{2 i-1}}{4 \times i}+\cdots\right. \\
& \left.\quad+\frac{C_{n}^{n-2}+C^{n-1}}{4 \times((n-1) / 2)}+\frac{C_{n}^{n}}{2 n+2}\right) \\
& =2^{n} \sum_{i=1}^{(n-1) / 2} \frac{C_{n}^{2 i-1}+C_{n}^{2 i}}{4 i}+\frac{2^{n-1}}{n+1}, \\
& \quad i=1,2, \ldots, \frac{n-1}{2} .
\end{aligned}
$$

The proof of Theorem 8 is completed.

3.2. The Kirchhoff Index in the Line Graph of Folded Hypercubes Networks $l\left(F Q_{n}\right)$. In the following theorem, we proposed the formula for calculating the Kirchhoff index, denoted by $\operatorname{Kf}\left(l\left(F Q_{n}\right)\right)$, on the line graph of folded hypercubes $l\left(F Q_{n}\right)$.

$$
\begin{gathered}
=2^{n} \sum_{i=1}^{n / 2} \frac{C_{n}^{n-i}+C_{n}^{n-i-1}}{4 i} \\
i=1,2, \ldots, \frac{n}{2} .
\end{gathered}
$$

Theorem 9. Let $l\left(F Q_{n}\right)$ be line graphs of hypercubes $F Q_{n}$ with any positive integer $n$; then

$$
\begin{gathered}
\text { (1) } \mathrm{Kf}\left(l\left(F Q_{n}\right)\right)=(n+1) 2^{n-1} \sum_{i=1}^{n / 2} \frac{C_{n}^{n-i}+C_{n}^{n-i-1}}{4 i} \\
+(n-1) 2^{2 n-3}, \\
i=1,2, \ldots, \frac{n}{2}, \text { if } n \equiv 0(\bmod 2) ; \\
\text { (2) } \mathrm{Kf}\left(l\left(F Q_{n}\right)\right)=(n+1) 2^{n-1} \sum_{i=1}^{(n-1) / 2} \frac{C_{n}^{2 i-1}+C_{n}^{2 i}}{4 i} \\
\quad i=1,2, \ldots, \frac{n-1}{2}, \text { if } n \equiv 1(\bmod 2),
\end{gathered}
$$

where the $4 i, i=1,2, \ldots,(n-1) / 2$ or $n / 2$ are the eigenvalues of the Laplacian matrix of folded hypercubes networks $F Q_{n}$ and $C_{n}^{i}$ are the binomial coefficients.

Proof. Now for convenience, we denote the numbers of vertices and edges in the folded hypercubes networks $F Q_{n}$ by $p=2^{n}$ and $q=(n+1) 2^{n-1}$, respectively.

By Lemma 4,

$$
P_{l\left(F Q_{n}\right)}(x)=(x-2 r)^{q-p} P_{F Q_{n}}(x) .
$$

Notice that folded hypercubes networks $F Q_{n}$ are regular graphs with the degree of any vertex being $r=n+1$; compare the spectrum of $F Q_{n}$ as follows. 
(1) If $n \equiv 0(\bmod 2)$,

$$
\operatorname{Spec}\left(F Q_{n}\right)=\left(\begin{array}{ccccccc}
0 & 4 & 8 & \cdots & 2 n-8 & 2 n-4 & 2 n \\
C_{n}^{n} & C_{n}^{n-1}+C_{n}^{n-2} & C_{n}^{n-3}+C_{n}^{n-4} & \cdots & C_{n}^{5}+C_{n}^{4} & C_{n}^{3}+C_{n}^{2} & C_{n}^{1}+C_{n}^{0}
\end{array}\right) .
$$

(2) If $n \equiv 1(\bmod 2)$,

$$
\operatorname{Spec}\left(F Q_{n}\right)=\left(\begin{array}{ccccccc}
0 & 4 & 8 & \cdots & 2 n-6 & 2 n-2 & 2 n+2 \\
C_{n}^{0} & C_{n}^{1}+C_{n}^{2} & C_{n}^{3}+C_{n}^{4} & \cdots & C_{n}^{n-4}+C_{n}^{n-3} & C_{n}^{n-2}+C_{n}^{n-1} & C_{n}^{n}
\end{array}\right) .
$$

We can easily obtain the spectrum of $l\left(F Q_{n}\right)$ as follows.

(1) If $n \equiv 0(\bmod 2)$,

$$
\operatorname{Spec}\left(l\left(F Q_{n}\right)\right)=\left(\begin{array}{cccccccc}
2 r & 0 & 4 & 8 & \cdots & 2 n-8 & 2 n-4 & 2 n \\
q-p & C_{n}^{n} & C_{n}^{n-1}+C_{n}^{n-2} & C_{n}^{n-3}+C_{n}^{n-4} & \cdots & C_{n}^{5}+C_{n}^{4} & C_{n}^{3}+C_{n}^{2} & C_{n}^{1}+C_{n}^{0}
\end{array}\right)
$$

(2) If $n \equiv 1(\bmod 2)$,

$$
\operatorname{Spec}\left(l\left(F Q_{n}\right)\right)=\left(\begin{array}{cccccccc}
2 r & 0 & 4 & 8 & \cdots & 2 n-6 & 2 n-2 & 2 n+2 \\
q-p & C_{n}^{0} & C_{n}^{1}+C_{n}^{2} & C_{n}^{3}+C_{n}^{4} & \cdots & C_{n}^{n-4}+C_{n}^{n-3} & C_{n}^{n-2}+C_{n}^{n-1} & C_{n}^{n}
\end{array}\right),
$$

where $\lambda_{0}, \lambda_{1}, \ldots, \lambda_{n}, \lambda_{n+1}, \ldots, \lambda_{2^{n}-1}$ are the eigenvalues of $F Q_{n}$ and $\lambda_{0} \leq \lambda_{1} \leq \lambda_{2} \leq \cdots \leq \lambda_{n} \leq \lambda_{n+1} \leq \cdots \leq \lambda_{2^{n}-1}$.

By Lemma 4 ,

$$
\begin{aligned}
\frac{\operatorname{Kf}\left(l\left(F Q_{n}\right)\right)}{(n+1) 2^{n-1}} & =\sum_{i=1}^{2^{n}-1} \frac{1}{\lambda_{i}}+\frac{(n+1) 2^{n-1}-2^{n}}{2 r} \\
& =\frac{\operatorname{Kf}\left(F Q_{n}\right)}{2^{n}}+\frac{(n+1) 2^{n-1}-2^{n}}{2 n+2} \\
& =\frac{K f\left(F Q_{n}\right)}{2^{n}}-\frac{2^{n-1}}{n+1}+2^{n-2} .
\end{aligned}
$$

Therefore, we clearly obtained

$$
\begin{aligned}
\mathrm{Kf}\left(l\left(F Q_{n}\right)\right) & =(n+1) 2^{n-1}\left(\frac{\mathrm{Kf}\left(F Q_{n}\right)}{2^{n}}-\frac{2^{n-1}}{n+1}+2^{n-2}\right) \\
& =\frac{n+1}{2} \mathrm{Kf}\left(F Q_{n}\right)+n 2^{2 n-3}-2^{2 n-3} \\
& =\frac{n+1}{2} \mathrm{Kf}\left(F Q_{n}\right)+(n-1) 2^{2 n-3} .
\end{aligned}
$$

Substituting the results of Theorem 8 into (25), we can get the formula for the Kirchhoff index on the line graph of folded hypercubes $\mathrm{Kf}\left(l\left(F Q_{n}\right)\right)$ :

$$
\begin{aligned}
& \text { (1) } \operatorname{Kf}\left(l\left(F Q_{n}\right)\right)=(n+1) 2^{n-1} \sum_{i=1}^{n / 2} \frac{C_{n}^{n-i}+C_{n}^{n-i-1}}{4 i} \\
& +(n-1) 2^{2 n-3}, \\
& i=1,2, \ldots, \frac{n}{2} \text {, if } n \equiv 0(\bmod 2) \text {; } \\
& \text { (2) } \mathrm{Kf}\left(l\left(F Q_{n}\right)\right)=(n+1) 2^{n-1} \sum_{i=1}^{(n-1) / 2} \frac{C_{n}^{2 i-1}+C_{n}^{2 i}}{4 i} \\
& +(n-1) 2^{2 n-3}+2^{n-2}, \\
& i=1,2, \ldots, \frac{n-1}{2} \text {, if } n \equiv 1(\bmod 2) \text {, }
\end{aligned}
$$

which completes the proof.

3.3. The Kirchhoff Index in the Subdivision Graph of Folded Hypercubes Networks $s\left(F Q_{n}\right)$. In an almost identical way as Theorem 9, we derived the formula for the Kirchhoff index on the subdivision graph of folded hypercubes $s\left(F Q_{n}\right)$, denoted by $\mathrm{Kf}\left(s\left(F Q_{n}\right)\right)$. 
Theorem 10. Let $s\left(F Q_{n}\right)$ be subdivision graphs of the folded hypercubes $F Q_{n}$ with any positive integer n; then,

(1) $\operatorname{Kf}\left(s\left(F Q_{n}\right)\right)=(n+3)^{2} 2^{n-1} \sum_{i=1}^{n / 2} \frac{C_{n}^{n-i}+C_{n}^{n-i-1}}{4 i}$

$$
\begin{aligned}
& +\frac{2^{n}+(n+1) 2^{n-1}}{n+3} \\
& +\frac{(n+1)^{2} 4^{n-1}-4^{n}}{2}, \\
& i=1,2, \ldots, \frac{n}{2}, \text { if } n \equiv 0(\bmod 2) ;
\end{aligned}
$$

(2) $\operatorname{Kf}\left(s\left(F Q_{n}\right)\right)=(n+3)^{2} 2^{n-1}$

$$
\begin{aligned}
& \times\left(\sum_{i=1}^{(n-1) / 2} \frac{C_{n}^{2 i-1}+C_{n}^{2 i}}{4 i}+\frac{2^{n-1}}{n+1}\right) \\
& +\frac{2^{n}+(n+1) 2^{n-1}}{n+3} \\
& +\frac{(n+1)^{2} 4^{n-1}-4^{n}}{2}, \\
& i=1,2, \ldots, \frac{n-1}{2}, \text { if } n \equiv 1(\bmod 2) .
\end{aligned}
$$

Proof. Now suppose that $P_{s\left(F Q_{n}\right)}(x)$ is the characteristic polynomial of the Laplacian matrix of the $s\left(F Q_{n}\right)$; let $P_{s\left(F Q_{n}\right)}(x)=$ $x^{2^{n}}+a_{1} x^{2^{n}-1}+a_{2} x^{2^{n}-2}+\cdots+a_{2^{n}-1} x$ and

$$
\operatorname{Spec}\left(s\left(F Q_{n}\right)\right)=\left\{\lambda_{0}, \lambda_{1}, \lambda_{2}, \ldots, \lambda_{n}, \lambda_{n+1}, \ldots, \lambda_{2^{n}-1}\right\},
$$

where $\lambda_{i}, i=0,1,2, \ldots, 2^{n}-1$, are the Laplacian eigenvalues of $s\left(F Q_{n}\right)$.

Then, by Lemma 7,

$$
\frac{\mathrm{Kf}\left(s\left(F Q_{n}\right)\right)}{2^{n}}=-\frac{a_{2^{n}-2}}{a_{2^{n}-1}} .
$$

By Lemma 5,

$$
\begin{aligned}
P_{s\left(F Q_{n}\right)}(x)=( & -1)^{2^{n}}(2-x)^{(n+1) 2^{n-1}-2^{n}} \\
\times & {\left[x^{2^{n}}(r+2-x)^{2^{n}}+a_{1} x^{2^{n}-1}(r+2-x)^{2^{n}-1}\right.} \\
& +\cdots+a_{2^{n}-2} x^{2}(r+2-x)^{2} \\
& \left.+a_{2^{n}-1} x(r+2-x)\right] .
\end{aligned}
$$

Consequently, the coefficient of $x^{2}$ in $P_{s\left(F Q_{n}\right)}(x)$ is

$$
\begin{gathered}
(-1)^{2^{n}}\left\{2^{(n+1) 2^{n-1}-2^{n}} a_{2^{n}-2}(r+2)^{2}\right. \\
-2^{(n+1) 2^{n-1}-2^{n}} a_{2^{n}-1}-\left[(n+1) 2^{n-1}-2^{n}\right] \\
\left.\times 2^{(n+1) 2^{n-1}-2^{n}-1}(r+2) a_{2^{n}-1}\right\},
\end{gathered}
$$

and the coefficient of $x$ in $P_{s\left(F Q_{n}\right)}(x)$ is

$$
(-1)^{2^{n}} 2^{(n+1) 2^{n-1}-2^{n}}(r+2) a_{2^{n}-1} .
$$

Since $s\left(F Q_{n}\right)$ has $2^{n}+(n+1) 2^{n-1}$ vertices. For convenience, denote $p=2^{n}, q=(n+1) 2^{n-1}$, and $r=n+1$, respectively. By Lemma 7 and substituting the coefficients into (12), we get

$$
\begin{aligned}
\frac{\mathrm{Kf}\left(s\left(F Q_{n}\right)\right)}{p+q}= & -\frac{2^{q-p}(r+2)^{2}-2^{q-p} a_{2^{n}-1}}{2^{q-p} a_{2^{n}-1}(r+2)} \\
& -\frac{(q-p) 2^{q-p-1} a_{2^{n}-1}(r+2)}{2^{q-p} a_{2^{n}-1}(r+2)} \\
= & -\frac{a_{2^{n}-2}(r+2)}{a_{2^{n}-1}}+\frac{1}{r+2}+\frac{q-p}{2} .
\end{aligned}
$$

Combining the results of Lemma 7 and (34),

$$
\frac{\mathrm{Kf}\left(s\left(F Q_{n}\right)\right)}{p+q}=(r+2) \frac{\mathrm{Kf}\left(F Q_{n}\right)}{2^{n}}+\frac{1}{r+2}+\frac{q-p}{2}
$$

Hence,

$$
\begin{aligned}
& \mathrm{Kf}\left(s\left(F Q_{n}\right)\right) \\
& \quad=(p+q)(r+2) \frac{\mathrm{Kf}\left(F Q_{n}\right)}{2^{n}}+\frac{p+q}{r+2}+\frac{q^{2}-p^{2}}{2} .
\end{aligned}
$$

Simplifying (36) by substituting $p=2^{n}, q=(n+1) 2^{n-1}$, and $r=n+1$, we can get

$$
\begin{aligned}
\mathrm{Kf}\left(s\left(F Q_{n}\right)\right) \\
=\left[2^{n}+(n+1) 2^{n-1}\right](n+3) \frac{\mathrm{Kf}\left(F Q_{n}\right)}{2^{n}} \\
\quad+\frac{2^{n}+(n+1) 2^{n-1}}{n+3}+\frac{(n+1)^{2} 4^{n-1}-4^{n}}{2} \\
=\frac{(n+3)^{2}}{2} \mathrm{Kf}\left(F Q_{n}\right)+\frac{2^{n}+(n+1) 2^{n-1}}{n+3} \\
\quad+\frac{(n+1)^{2} 4^{n-1}-4^{n}}{2} .
\end{aligned}
$$


Note that the results of Theorem 8 and (37), we can get the formulae for the Kirchhoff index of the subdivision graph of the folded hypercubes $\operatorname{Kf}\left(s\left(F Q_{n}\right)\right)$ :

$$
\begin{aligned}
\text { (1) } \mathrm{Kf}\left(s\left(F Q_{n}\right)\right)= & (n+3)^{2} 2^{n-1} \sum_{i=1}^{n / 2} \frac{C_{n}^{n-i}+C_{n}^{n-i-1}}{4 i} \\
+ & \frac{2^{n}+(n+1) 2^{n-1}}{n+3}+\frac{(n+1)^{2} 4^{n-1}-4^{n}}{2}, \\
& i=1,2, \ldots, \frac{n}{2}, \text { if } n \equiv 0(\bmod 2) ;
\end{aligned}
$$

(2) $\mathrm{Kf}\left(s\left(F Q_{n}\right)\right)=(n+3)^{2} 2^{n-1}$

$$
\begin{gathered}
\times\left(\sum_{i=1}^{(n-1) / 2} \frac{C_{n}^{2 i-1}+C_{n}^{2 i}}{4 i}+\frac{2^{n-1}}{n+1}\right) \\
+\frac{2^{n}+(n+1) 2^{n-1}}{n+3}+\frac{(n+1)^{2} 4^{n-1}-4^{n}}{2}, \\
\quad i=1,2, \ldots, \frac{n-1}{2}, \text { if } n \equiv 1(\bmod 2),
\end{gathered}
$$

which completes the proof.

3.4. The Kirchhoff Index in the Total Graph of Folded Hypercubes Networks $t\left(F Q_{n}\right)$. We now proved the formula for the Kirchhoff index in the total graph of the folded hypercubes $t\left(F Q_{n}\right)$, denoted by $\mathrm{Kf}\left(t\left(F Q_{n}\right)\right)$.

Theorem 11. Let $t\left(F Q_{n}\right)$ be the total graphs of the folded hypercubes networks $F Q_{n}$ with any positive integer n; then,

(1) $\mathrm{Kf}\left(t\left(F Q_{n}\right)\right)$

$$
\begin{gathered}
=\frac{2^{n-1}(n+3)^{2}}{n+4} \sum_{i=1}^{n / 2} \frac{C_{n}^{n-i}+C_{n}^{n-i-1}}{4 i} \\
+\frac{2^{2 n-3}(n-1)(n+3)}{n+2}+2^{n-1} \\
+\frac{2^{n-1}(n+3)(n+5)}{n+4} \sum_{i=1}^{n / 2} \frac{C_{n}^{n-i}+C_{n}^{n-i-1}}{4 i+n+4}, \\
\quad i=1,2, \ldots, \frac{n}{2}, \text { if } n \equiv 0(\bmod 2) ;
\end{gathered}
$$

(2) $\mathrm{Kf}\left(t\left(F Q_{n}\right)\right)$

$$
\begin{aligned}
= & \frac{2^{n-1}(n+3)^{2}}{n+4} \\
& \times\left(\sum_{i=1}^{(n-1) / 2} \frac{C_{n}^{2 i-1}+C_{n}^{2 i}}{4 i}+\frac{1}{2 n+2}\right)
\end{aligned}
$$

$$
\begin{aligned}
& +\frac{2^{2 n-3}(n-1)(n+3)}{n+2}+2^{n-1} \\
& +\frac{2^{n-1}(n+3)(n+5)}{n+4} \sum_{i=1}^{(n-1) / 2} \frac{C_{n}^{2 i-1}+C_{n}^{2 i}}{4 i+n+4}, \\
& i=1,2, \ldots, \frac{n-1}{2}, \text { if } n \equiv 1(\bmod 2),
\end{aligned}
$$

where the $4 i, i=1,2, \ldots,(n-1) / 2$ or $n / 2$, are the eigenvalues of the Laplacian matrix of folded hypercubes networks $F Q_{n}$ and $C_{n}^{i}$ are the binomial coefficients.

Proof. Let $P_{t\left(F Q_{n}\right)}(x)=x^{2^{n}}+a_{1} x^{2^{n}-1}+a_{2} x^{2^{n}-2}+\cdots+a_{2^{n}-1} x$ and

$$
\operatorname{Spec}\left(t\left(F Q_{n}\right)\right)=\left\{\lambda_{0}, \lambda_{1}, \lambda_{2}, \ldots \lambda_{n}, \lambda_{n+1}, \ldots, \lambda_{2^{n}-1}\right\},
$$

where $\lambda_{i}, i=0,1,2, \ldots, 2^{n}-1$, are the Laplacian eigenvalues of $F Q_{n}$.

Applying Lemma 5, the Laplacian characteristic polynomial of $t\left(F Q_{n}\right)$ is

$$
\begin{aligned}
P_{t\left(F Q_{n}\right)}(x)= & x(x-n-3)(x-2 n-4)^{q-p} \\
& \times \prod_{i=1}^{2^{n}-1}\left\{\left[x^{2}-2 x-(n+1) x\right]\right. \\
& \left.+[3-2 x+(n+1)] \lambda_{i}+\lambda_{i}^{2}\right\} \\
= & x(x-n-3)(x-2 n-4)^{q-p} \\
& \times \prod_{i=1}^{2^{n}-1}\left[x^{2}-\left(3+n+2 \lambda_{i}\right) x+\lambda_{i}^{2}+(n+4) \lambda_{i}\right] .
\end{aligned}
$$

Notice that $t\left(F Q_{n}\right)$ has $2^{n}+(n+1) 2^{n-1}$ vertices, that is, $p+q$ vertices. By Lemma 6 , we can get the following:

$$
\begin{aligned}
\mathrm{Kf}\left(t\left(F Q_{n}\right)\right)= & \frac{(r+2)^{2}}{2(r+3)} \mathrm{Kf}\left(F Q_{n}\right)+\frac{n^{2}\left(r^{2}-4\right)}{8(r+1)}+\frac{n}{2} \\
& +\frac{n(r+2)(r+4)^{2^{n}-1}}{2(r+3)} \sum_{i=1}^{n} \frac{1}{\lambda_{i}+3+r} .
\end{aligned}
$$


Consider that $F Q_{n}$ has $2^{n}$ vertices and $r=n+1$, we have

$$
\begin{aligned}
\mathrm{Kf}\left(t\left(F Q_{n}\right)\right)= & \frac{(n+3)^{2}}{2(n+4)} \mathrm{Kf}\left(F Q_{n}\right) \\
& +\frac{4^{n}\left[(n+1)^{2}-4\right]}{8(n+2)}+2^{n-1} \\
& +\frac{2^{n}(n+3)(n+5)^{2^{n}-1}}{2(n+4)} \sum_{i=1} \frac{1}{\lambda_{i}+n+4} \\
= & \frac{(n+3)^{2}}{2(n+4)} \mathrm{Kf}\left(F Q_{n}\right) \\
& +\frac{2^{2 n-3}(n-1)(n+3)}{n+2}+2^{n-1} \\
& +\frac{2^{n-1}(n+3)(n+5)^{2^{n}-1}}{n+4} \sum_{i=1}^{\frac{1}{\lambda_{i}+n+4}} .
\end{aligned}
$$

From (43), the relationships between the folded hypercubes networks $F Q_{n}$ and its variant networks $t\left(F Q_{n}\right)$ for Kirchhoff index are as follows:

$$
\begin{aligned}
\mathrm{Kf}\left(t\left(F Q_{n}\right)\right)= & \frac{(n+3)^{2}}{2(n+4)} \mathrm{Kf}\left(F Q_{n}\right) \\
& +\frac{2^{2 n-3}(n-1)(n+3)}{n+2}+2^{n-1} \\
& +\frac{2^{n-1}(n+3)(n+5)^{2^{n}-1}}{n+4} \sum_{i=1}^{n} \frac{1}{\lambda_{i}+n+4} .
\end{aligned}
$$

Substituting results of Theorem 8 into (44) and simplifying (44), we can get the formula for the Kirchhoff index on the total graph of folded hypercubes $\operatorname{Kf}\left(t\left(F Q_{n}\right)\right)$; we consider two cases in terms of if $n \equiv 0(\bmod 2)$ and if $n \equiv 1(\bmod 2)$,

(1) $\mathrm{Kf}\left(t\left(F Q_{n}\right)\right)=\frac{2^{n-1}(n+3)^{2}}{n+4} \sum_{i=1}^{n / 2} \frac{C_{n}^{n-i}+C_{n}^{n-i-1}}{4 i}$

$$
\begin{aligned}
& +\frac{2^{2 n-3}(n-1)(n+3)}{n+2}+2^{n-1} \\
& +\frac{2^{n-1}(n+3)(n+5)}{n+4} \sum_{i=1}^{n / 2} \frac{C_{n}^{n-i}+C_{n}^{n-i-1}}{4 i+n+4}, \\
& i=1,2, \ldots, \frac{n}{2}, \text { if } n \equiv 0(\bmod 2) ;
\end{aligned}
$$

(2) $\mathrm{Kf}\left(t\left(F Q_{n}\right)\right)=\frac{2^{n-1}(n+3)^{2}}{n+4}$

$$
\begin{aligned}
& \times\left(\sum_{i=1}^{(n-1) / 2} \frac{C_{n}^{2 i-1}+C_{n}^{2 i}}{4 i}+\frac{1}{2 n+2}\right) \\
& +\frac{2^{2 n-3}(n-1)(n+3)}{n+2}+2^{n-1} \\
& +\frac{2^{n-1}(n+3)(n+5)}{n+4} \sum_{i=1}^{(n-1) / 2} \frac{C_{n}^{2 i-1}+C_{n}^{2 i}}{4 i+n+4} \\
& i=1,2, \ldots, \frac{n-1}{2}, \text { if } n \equiv 1(\bmod 2),
\end{aligned}
$$

where the $4 i, i=1,2, \ldots,(n-1) / 2$ or $n / 2$, are the eigenvalues of the Laplacian matrix of folded hypercubes networks $F Q_{n}$ and $C_{n}^{i}$ are the binomial coefficients.

This completes the proof of Theorem 11 .

Remark 12. The authors [27] gave the formulae for the Kirchhoff index of $l(G), s(G)$, and $t(G)$, where $G$ is a regular graph. In this paper, we proposed the special formulae for calculating the Kirchhoff indexes of the folded hypercubes networks $F Q_{n}$ and its three variant networks $l\left(F Q_{n}\right), s\left(F Q_{n}\right)$, and $t\left(F Q_{n}\right)$, respectively, which enhanced related results on the Kirchhoff index.

\section{Conclusions}

The main purpose of this paper is to investigate the Kirchhoff index of the folded hypercubes networks and related networks, which are important networks topology indexes for parallel processing computer systems. We established the relationships between the folded hypercubes networks $F Q_{n}$ and its three variant networks $l\left(F Q_{n}\right), s\left(F Q_{n}\right)$, and $t\left(F Q_{n}\right)$ for their Kirchhoff index, respectively, by deducing the characteristic polynomial of the Laplacian matrix related networks.

Moreover, the explicit formulae were proposed for calculating the Kirchhoff indexes of $F Q_{n}, l\left(F Q_{n}\right), s\left(F Q_{n}\right)$, and $t\left(F Q_{n}\right)$, respectively, by making use of the characteristic polynomial of the Laplacian matrix in spectral graph theory and Vieta's theorem.

\section{Conflict of Interests}

The authors declare that there is no conflict of interests regarding the publication of this paper.

\section{Acknowledgments}

The work of Jinde Cao was funded by the National Natural Science Foundation of China under Grant 61272530, the Natural Science Foundation of Jiangsu Province of China under Grant BK2012741, the Specialized Research Fund for the Doctoral Program of Higher Education under Grant 20110092110017. The work of Jiabao Liu was supported by 
the Natural Science Foundation of Anhui Province of China under Grant no. KJ2013B105. The work of Xiangfeng Pan was supported by the National Science Foundation of China under Grant nos. 11171097 and 11371028.

\section{References}

[1] J. M. Xu, Topological Strucure and Analysis of Interconnction Networks, Kluwer Academic publishers, Dordrcht, The Netherlands, 2001.

[2] D. J. Klein and M. Randić, "Resistance distance," Journal of Mathematical Chemistry, vol. 12, no. 1-4, pp. 81-95, 1993.

[3] B. Zhou and N. Trinajstić, "A note on Kirchhoff index," Chemical Physics Letters, vol. 455, no. 1-3, pp. 120-123, 2008.

[4] E. Estrada and N. Hatano, "Topological atomic displacements, Kirchhoff and Wiener indices of molecules," Chemical Physics Letters, vol. 486, no. 4-6, pp. 166-170, 2010.

[5] A. D. Maden, A. S. Cevik, I. N. Cangul, and K. C. Das, "On the Kirchhoff matrix, a new Kirchhoff index and the Kirchhoff energy," Journal of Inequalities and Applications, p. 2013:337, 2013.

[6] H. Zhang, X. Jiang, and Y. Yang, "Bicyclic graphs with extremal Kirchhoff index," Communications in Mathematical and in Computer Chemistry, vol. 61, no. 3, pp. 697-712, 2009.

[7] H. Y. Zhu, D. J. Klein, and I. Lukovits, "Extensions of the Wiener number," Journal of Chemical Information and Computer Sciences, vol. 36, no. 3, pp. 420-428, 1996.

[8] I. Gutman and B. Mohar, "The quasi-Wiener and the Kirchhoff indices coincide," Journal of Chemical Information and Computer Sciences, vol. 36, no. 5, pp. 982-985, 1996.

[9] W. Xiao and I. Gutman, "Resistance distance and Laplacian spectrum," Theoretical Chemistry Accounts, vol. 110, no. 4, pp. 284-289, 2003.

[10] D. Babić, D. J. Klein, I. Lukovits, S. Nikolić, and N. Trinajstić, "Resistance-distance matrix: a computational algorithm and its applications," International Journal of Quantum Chemistry, vol. 90, no. 1, pp. 166-176, 2002.

[11] J. L. Palacios and J. M. Renom, "Bounds for the Kirchhoff index of regular graphs via the spectra of their random walks," International Journal of Quantum Chemistry, vol. 110, no. 9, pp. 1637-1641, 2010.

[12] I. Lukovits, S. Nikolić, and N. Trinajstić, "Resistance distance in regular graphs," International Journal of Quantum Chemistry, vol. 71, no. 3, pp. 217-225, 1999.

[13] J. L. Palacios, "Closed-form formulas for Kirchhoff index," International Journal of Quantum Chemistry, vol. 81, no. 2, pp. 135-140, 2001.

[14] H. Zhang, Y. Yang, and C. Li, "Kirchhoff index of composite graphs," Discrete Applied Mathematics, vol. 157, no. 13, pp. 29182927, 2009.

[15] C. Arauz, "The Kirchhoff indexes of some composite networks," Discrete Applied Mathematics, vol. 160, no. 10-11, pp. 1429-1440, 2012.

[16] M. Bianchi, A. Cornaro, J. L. Palacios, and A. Torriero, "Bounds for the Kirchhoff index via majorization techniques," Journal of Mathematical Chemistry, vol. 51, no. 2, pp. 569-587, 2013.

[17] H. Wang, H. Hua, and D. Wang, "Cacti with minimum, secondminimum, and third-minimum Kirchhoff indices," Mathematical Communications, vol. 15, no. 2, pp. 347-358, 2010.
[18] A. El-Amawy and S. Latifi, "Properties and performance of folded hypercubes," IEEE Transactions on Parallel and Distributed Systems, vol. 2, no. 1, pp. 31-42, 1991.

[19] J. Fink, "Perfect matchings extend to Hamilton cycles in hypercubes," Journal of Combinatorial Theory B, vol. 97, no. 6, pp. 1074-1076, 2007.

[20] J. Liu, J. Cao, X. Pan, and A. Elaiw, "The Kirchhoff index of hypercubes and related complex networks," Discrete Dynamics in Nature and Society, vol. 2013, Article ID 543189, 7 pages, 2013.

[21] M. Chen and B. X. Chen, "Spectra of folded hypercubes," Journal of East China Normal University Nature Science, vol. 2, no. 39, pp. 39-46, 2011.

[22] X. He, H. Liu, and Q. Liu, "Cycle embedding in faulty folded hypercube," International Journal of Applied Mathematics \& Statistics, vol. 37, no. 7, pp. 97-109, 2013.

[23] S. Cao, H. Liu, and X. He, "On constraint fault-free cycles in folded hypercube," International Journal of Applied Mathematics and Statistics, vol. 42, no. 12, pp. 38-44, 2013.

[24] Y. Zhang, H. Liu, and M. Liu, "Cycles embedding on folded hypercubes with vertex faults," International Journal of Applied Mathematics \& Statistics, vol. 41, no. 11, pp. 58-70, 2013.

[25] X. B. Chen, "Construction of optimal independent spanning trees on folded hypercubes," Information Sciences, vol. 253, pp. 147-156, 2013.

[26] M. Chen, X. Guo, and S. Zhai, "Total chromatic number of folded hypercubes," Ars Combinatoria, vol. 111, pp. 265-272, 2013.

[27] X. Gao, Y. Luo, and W. Liu, "Kirchhoff index in line, subdivision and total graphs of a regular graph," Discrete Applied Mathematics, vol. 160, no. 4-5, pp. 560-565, 2012.

[28] Z. You, L. You, and W. Hong, "Comment on "Kirchhoff index in line, subdivision and total graphs of a regular graph"', Discrete Applied Mathematics, vol. 161, no. 18, pp. 3100-3103, 2013. 


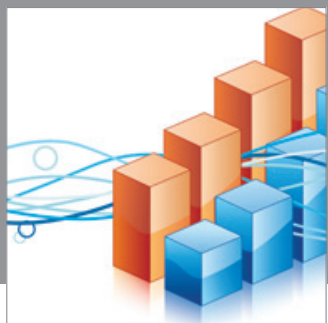

Advances in

Operations Research

mansans

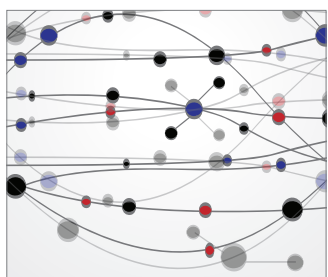

The Scientific World Journal
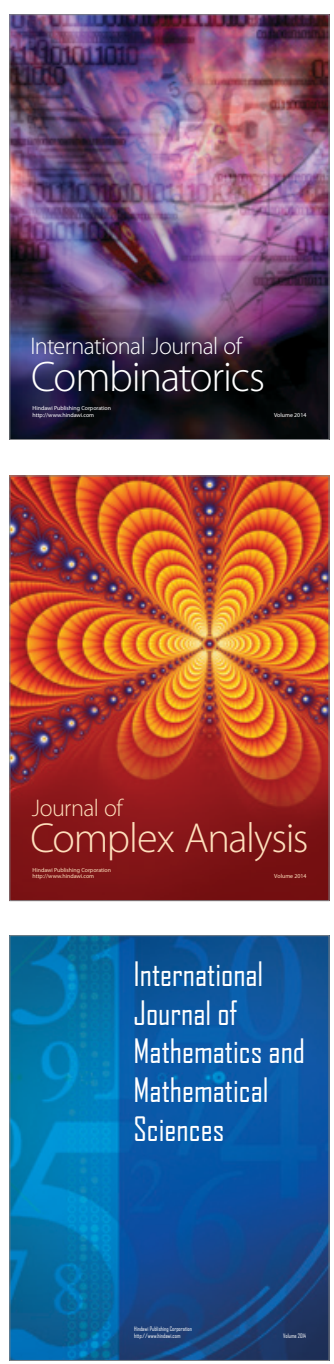
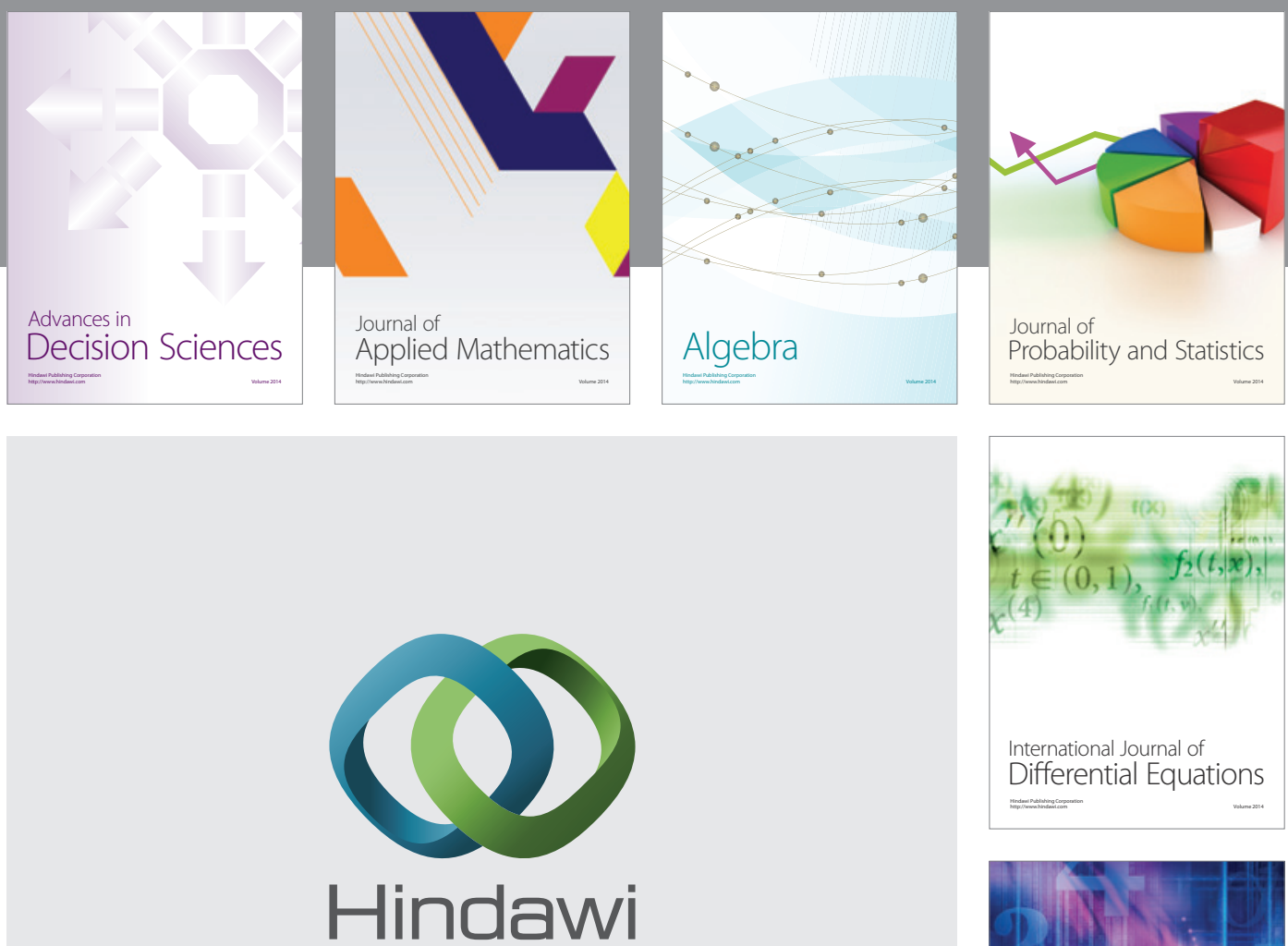

Submit your manuscripts at http://www.hindawi.com
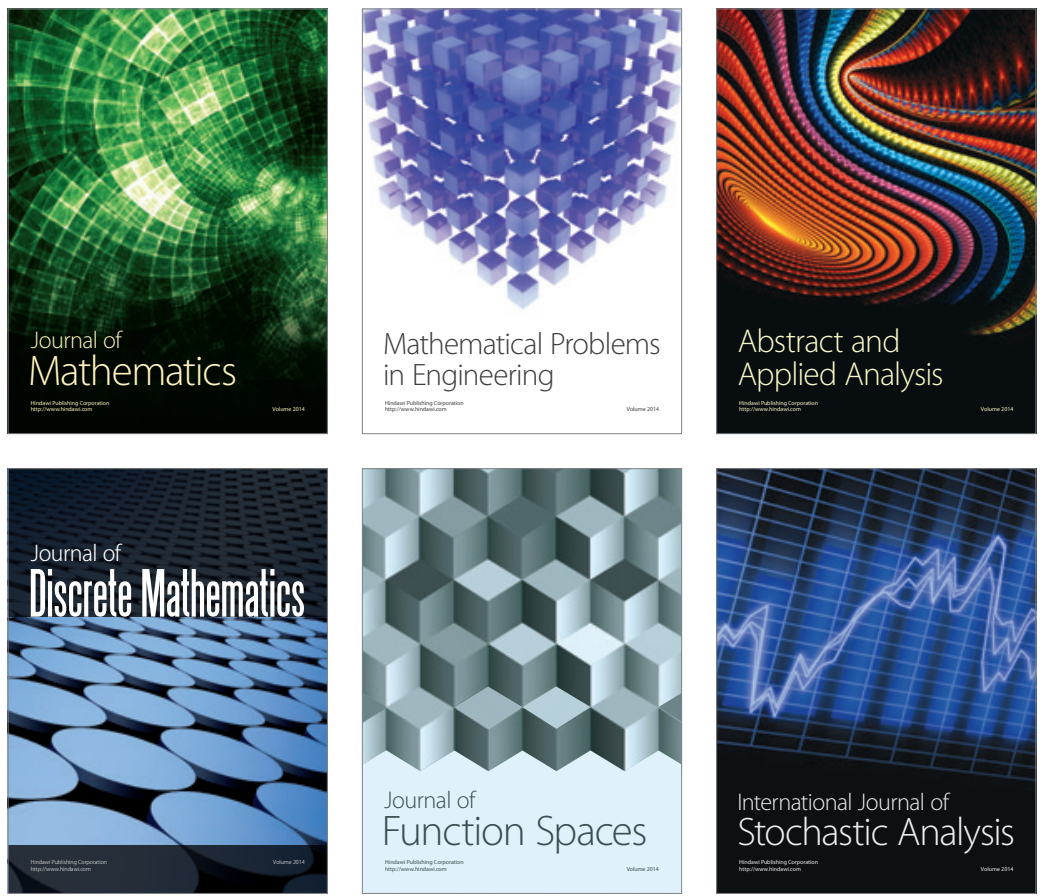

Journal of

Function Spaces

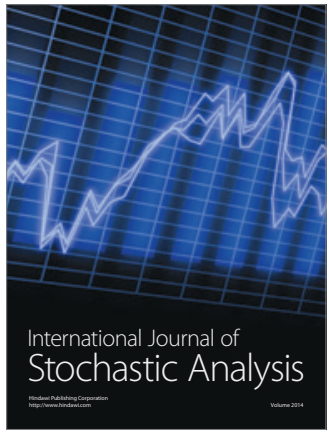

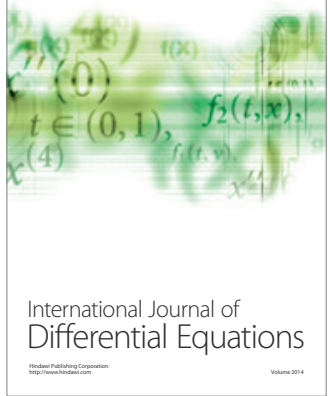
